\title{
Nuclear activity in nearby galaxies ${ }^{\star}$ Mid-infrared imaging with the VLT
}

\author{
R. Siebenmorgen ${ }^{1}$, M. Haas ${ }^{2}$, E. Pantin ${ }^{3}$, E. Krügel ${ }^{4}$, C. Leipski ${ }^{5}$, H. U. Käufl ${ }^{1}$, \\ P. O. Lagage ${ }^{3}$, A. Moorwood ${ }^{1}$, A. Smette ${ }^{6}$, and M. Sterzik ${ }^{6}$
}

1 European Southern Observatory, Karl Schwarzschild Strasse 2, 85748 Garching b. München, Germany e-mail: rsiebenm@eso.org

2 Astronomisches Institut, Ruhr-Universität Bochum, Universitätstr. 150, NA7, 44801 Bochum, Germany

3 UMR7158 CEA-CNRS-U. Paris 7, DSM/DAPNIA/Service d'Astrophysique, CEA/Saclay, 91191 Gif-sur-Yvette, France

4 Max-Planck-Institut for Radioastronomie, Auf dem Hügel 69, Postfach 2024, 53010 Bonn, Germany

5 Department of Physics, University of California, Santa Barbara, CA 93106, USA

${ }^{6}$ European Southern Observatory, Casilla 19001, Santiago 19, Chile

Received 8 February 2008 / Accepted 19 May 2008

\section{ABSTRACT}

\begin{abstract}
Aims. Dust enshrouded activity can be studied ideally by mid-infrared (MIR) observations. To explore the active galactic nuclei (AGN) versus star forming origin of the nuclear MIR emission of galaxies, observations of high spatial resolution are required. We report on $11.3 \mu \mathrm{m}$ observations with VISIR at the VLT, which reach $0.35^{\prime \prime}$ spatial resolution (FWHM).

Methods. During the scientific verification of VISIR, we observed a sample of 36 nearby galaxies with a range of optically classified nuclear activity: 17 black hole driven AGN, 10 starbursts (SB), and 9 quiescent spirals.

Results. 16 objects out of 17 AGN are detected and unresolved, 5 out of 10 SB are detected and resolved with structured emission up to a few arcsec, while for 5 targets out of $10 \mathrm{SB}$ and all 9 quiet nuclei low upper limits are provided. The morphology of the resolved SB nuclei is similar to that observed at radio frequencies. The compactness of AGN and the extent of SB nuclei is consistent with the predictions of radiative transfer models and Spitzer spectra observed at much lower spatial resolution than with VISIR. We introduce the nuclear MIR surface brightness, $S$, as a quantitative measurement for AGN and SB activity. Although one is unable to distinguish between AGN and SB activity using the nuclear MIR surface brightness derived from $4 \mathrm{~m}$ class telescopes, our data indicate that, out to a distance of $100 \mathrm{Mpc}$, the MIR surface brightness acquired by $8 \mathrm{~m}$ class telescopes can differentiate AGN from SB behavior.
\end{abstract}

Key words. infrared: galaxies - galaxies: nuclei - galaxies: active - galaxies: spiral - galaxies: starburst

\section{Introduction}

The mid infrared (MIR) luminosity of the central region of galaxies has long been known to be a reliable indicator of activity much less affected by extinction than optical and NIR observations (Rieke \& Lebofsky 1978). The MIR emission traces thermal radiation from hot dust $(\sim 100 \mathrm{~K})$ heated either by OB stars or black hole activity. For quasars, the presence of a non-thermal synchrotron component is also conceivable. Based on data from the Infrared Space Observatory and the Spitzer Space Telescope, many diagnostics have been proposed to quantify which of the two types of activity, AGN or SB, dominates. The diagrams include the slope of the IR continuum, the strength of the PAH features, the ratio of high to low ionization lines and the silicate band (Genzel et al. 1998; Laurent et al. 2000; Sturm et al. 2002; Siebenmorgen et al. 2005; Hao et al. 2005; Sturm et al. 2006; Spoon et al. 2007). However, given the small sizes of these telescopes in space, the diagnostics may be contaminated by the contribution of the surrounding galaxy. To assess the origin of the nuclear MIR luminosity, high spatial resolution observations from larger ground-based telescopes are required.

Numerous MIR studies have been undertaken to compare the compactness of AGN and SB. While observations with $4 \mathrm{~m}$

^ Based on ESO: 68.B-0066(A) and 60.A-9244(A). class telescopes have been performed for large samples, the spatial resolution and sensitivity limited these studies to a comparison between central $\left(1^{\prime \prime}-5^{\prime \prime}\right)$ and extended MIR emission; AGN were found to be more compact than SB (e.g. Roche et al. 1991; Maiolino et al. 1995; Gorjian et al. 2004), and only a few nearby SB could be resolved in some detail (e.g. Siebenmorgen et al. 2004; Galliano et al. 2005a using TIMMI2 at the ESO $3.6 \mathrm{~m}$ telescope). With the advent of MIR facilities at $10 \mathrm{~m}$ class telescopes, a small number of nearby, mostly (ultra)-luminous infrared galaxies have been observed so far, successfully resolving some SB (Soifer et al. 1999, 2000, 2001 with Keck/MIRLIN, Alonso-Herrero et al. 2006 with Gemini/T-ReCS, Galliano et al. 2005b; Wold et al. 2006 with VLT/VISIR).

Here we report on the science verification results from VISIR, the MIR instrument at the VLT (Lagage et al. 2004). We exploit VISIR's capabilities to study a sample of bright, nearby spirals and compare the observations with both radiative transfer models and radio-interferometric maps.

\section{VISIR science verification observations}

A sample of 36 targets with distance $<100 \mathrm{Mpc}$ were selected from the catalog of spiral galaxies by Albrecht et al. (2007). This catalog is a complete FIR-selected sample with $S_{100 \mu \mathrm{m}}>9 \mathrm{Jy}$ and includes 168 objects whose $\mathrm{CO}$ gas emission and submm 
Table 1. Observed sample sorted by AGN, starbursts and normal galaxies. Specified is morphological type (NED), optical classification and distance $D$ (Albrecht et al. 2007). VISIR parameters are: observing date, integration time, $t_{\text {int }}$, and integrated flux or $3 \sigma$ upper limit in filter PAH2, $F_{11.25 \mu \mathrm{m}}$, and for resolved sources the area, $A$, of the MIR emission, respectively.

\begin{tabular}{|c|c|c|c|c|c|c|c|}
\hline Name & type & "activity & $\begin{array}{c}D \\
(\mathrm{Mpc})\end{array}$ & $\overline{\text { date }}$ & $\begin{array}{l}t_{\text {int }} \\
\text { (s) }\end{array}$ & $\begin{array}{r}F_{11.2 \mu \mu \mathrm{m}} \\
(\mathrm{mJy})\end{array}$ & $\begin{array}{c}A \\
\left(\mathrm{pc}^{2}\right)\end{array}$ \\
\hline $\begin{array}{l}\text { AGN: } \\
\text { NGC } 4418\end{array}$ & $\mathrm{SAB}(\mathrm{s}) \mathrm{a}$ & Sy2 & 28.3 & 2005-01-31 & 906 & 110 & \\
\hline \multicolumn{8}{|l|}{ Starburst: } \\
\hline ESO091-G016 & $\mathrm{Sb}$ & ? & 26.7 & 2005-02-02 & 1236 & $<1.6$ & \\
\hline ESO126-G002 & $\mathrm{SB}(\mathrm{rs}) \mathrm{ab}$ & $\mathrm{HII}$ & 38.5 & 2005-01-29 & 1413 & 305 & 325 \\
\hline ESO163-G011 & $\mathrm{SB}(\mathrm{s}) \mathrm{bsp}$ & ? & 37.3 & 2005-01-29 & 707 & $<1.6$ & \\
\hline ESO500-G034 & $\mathrm{SB}(\mathrm{s}) 0 / \mathrm{a}$ & HII & 48.8 & 2005-01-27 & 1766 & 53 & 380 \\
\hline Mkn 617 & $\mathrm{SB}(\mathrm{s}) \mathrm{c}$ pec & HII & 62.8 & $2004-10-02$ & 1843 & 720 & 465 \\
\hline Mkn 708 & $\mathrm{SBc}$ & HII & 25.2 & 2005-01-29 & 1236 & 350 & 123 \\
\hline Mkn 708 (core) & & & & & & 20 & \\
\hline Mkn 1022 & SA pec & SB & 51.2 & 2004-10-02 & 2089 & $<0.9$ & \\
\hline NGC 1482 & $\mathrm{SA} 0+$ pec & HII & 25.2 & 2004-09-25 & 1186 & 510 & 471 \\
\hline NGC 5258 & SA(s)b pec & HII,LINER & 90.3 & 2005-02-02 & 707 & $<2.2$ & \\
\hline NGC 5786 & $\mathrm{SAB}(\mathrm{s}) \mathrm{bc}$ & $?$ & 39.2 & 2005-02-03 & 538 & $<3.3$ & \\
\hline \multicolumn{8}{|l|}{ Normal: } \\
\hline ESO061-G011 & $\mathrm{S}$ & & 81.3 & 2005-01-29 & 707 & $<1.3$ & \\
\hline ESO093-G003 & $\mathrm{SAB}(\mathrm{r}) 0 / \mathrm{a}$ & & 24.5 & 2005-02-01 & 883 & $<1.6$ & \\
\hline ESO317-G023 & $\mathrm{SB}(\mathrm{rs}) \mathrm{a}$ & & 35.8 & 2005-02-01 & 707 & $<1.8$ & \\
\hline ESO493-G016 & $\mathrm{Sb}-\mathrm{c}$ & & 34.7 & 2005-01-31 & 707 & $<1.6$ & \\
\hline NGC 2706 & Sbc? sp & & 21.3 & 2005-01-31 & 1060 & $<1.3$ & \\
\hline NGC 3318 & $\mathrm{SA}(\mathrm{sr}) \mathrm{b}$ & & 37.2 & 2005-01-31 & 1766 & $<1.0$ & \\
\hline NGC 3366 & $\mathrm{SB}(\mathrm{rs}) \mathrm{bc}$ & & 38.1 & 2005-02-01 & 707 & $<1.8$ & \\
\hline NGC 4746 & Sb sp & & 23.7 & 2005-01-31 & 883 & $<1.4$ & \\
\hline NGC 4900 & $\mathrm{SB}(\mathrm{rs}) \mathrm{c}$ & & 12.0 & 2005-02-02 & 707 & $<2.2$ & \\
\hline
\end{tabular}

emission is well studied. It is based on work carried out by Krügel et al. (1990), Chini et al. (1992), and Chini et al. (1995). Following the classification from optical spectroscopy as listed by Albrecht et al. (2007), our sample consists of: i) seventeen AGN; ii) ten starbursts and iii) nine sources classified as normal, inactive spirals; their gas mass is comparable to that of the active galaxies in the sample. The median distances of the three sub-samples are similar and between $35-40 \mathrm{Mpc}$. The motivation for including the inactive spirals was to search for faint compact MIR emission from low-luminosity nuclear starbursts, which may so far have escaped detection in low-resolution observations.

Imaging data through filter PAH2 $(11.25 \pm 0.6 \mu \mathrm{m})$ were obtained under good and stable weather conditions during VISIR science verification in Oct. 2004-Feb. 2005. During the observations the optical seeing was $\lesssim 1^{\prime \prime}$ and air mass $\lesssim 1.4$. The $0.127^{\prime \prime}$ pixel scale, corresponding to a $32^{\prime \prime} \times 32^{\prime \prime}$ field of view, was used. Thermal background subtraction were performed with on-array chopping and nodding amplitudes of $16^{\prime \prime}$ each. When a target could not be identified in the real time display after $15 \mathrm{~min}$ integration, we switched to another object; otherwise, typical integration times were $25 \mathrm{~min}$.

VISIR images sometimes exhibit stripes that are generated by a few high gain detector pixels. They are removed by dedicated reduction methods (Pantin et al. 2005). The large number of bad pixels prevents us from deconvolving the images, but noise filtering can be applied using a wavelet technique described by Starck \& Murtagh (2002). In the final images, unresolved sources have a FWHM of $0.35^{\prime \prime}$. Measurements of photometric standard stars were assigned to each set of science observations and used as PSF reference. Photometry was derived from multi-aperture analysis of the final image. The absolute photometric uncertainty that we achieved is below $10 \%$. This error estimate was based on internal consistency and monitoring of the calibration observations. Haas et al. (2007) found good consistency between VISIR $11.25 \mu$ m photometry of 16 Seyferts and other published measurements. In the VISIR observations, we achieved a $3 \sigma$ point source detection limit of $\sim 4 \mathrm{mJy}$ following a $10 \mathrm{~min}$. integration. The differential observing technique prevents to detect smooth extended structure of more than $8^{\prime \prime}$, which is half the chopping and nodding amplitude. The observations are summarized in Table 1, where targets are sorted by type, morphological, and optical classification. We also provide VISIR $11.25 \mu \mathrm{m}$ photometry, resolved area, distance, observing date, and total integration time. With the exception of NGC 4418, the AGN observations are listed in a companion paper (Haas et al. 2007).

All of the AGN, apart from one, are unresolved. The nondetection of NGC 5427 may be due to chopping in a structurerich emission as discussed by Haas et al. (2007); this source is excluded from the following discussion. Five out of ten starbursts show extended emission on scales of several arcsec. Low upper limits could be achieved for the remaining 5 galaxies classified as SB and the 9 quiet spirals. Obviously, these galaxies do not have striking hot dust emission heated by nuclear activity. For 3 out of 5 undetected SB, we note that no entry exists in NED indicating enhanced activity, thus they may be quiet.

\section{MIR morphology of the galactic nucleus}

The MIR luminosity of galactic nuclei often originates from a composite of starburst and AGN activity. Observations have implied that the extent of the nuclear MIR emission may be used as an indicator to distinguish between both activity types. Radiative transfer models (e.g. Siebenmorgen et al. 2004) provide quantitative predictions supporting this hypothesis. Here, we compare 
VISIR observations with both literature data and model predictions. For resolved sources we check how well the MIR - radio correlation holds.

\subsection{AGN - core dominated}

At 1.5" resolution, Gorjian et al. (2004) detected 62 Seyfert galaxies in the MIR. All detected sources corresponded to a central point source; extended structure was observed in: i) Arp 220, a well-known merger with starburst activity (e.g. Soifer et al. 1999; Spoon et al. 2004); ii) NGC 7469, a composite object with a Seyfert I nucleus and a circumnuclear starburst ring (Krabbe et al. 2001); iii) NGC 1068, where a set of clumps are located in the narrow line region and not associated with the torus (Galliano et al. 2005b); and iv) Mrk1239, where the faint MIR extension claimed by Gorjian et al. (2004) cannot be confirmed by our short exposures. It is unresolved in VLA maps (Thean et al. 2000) and slightly extended in [OIII] (Mulchaey et al. 1996). At 0.7" resolution with TIMMI2, Siebenmorgen et al. (2004) detected 15 Sy galaxies in the MIR. Their emission is dominated by an unresolved core and for some Seyferts (Cen A (Radomski et al. 2008), Circinus (Roche et al. 2006), NGC 1365, NGC 1386, NGC 4388, NGC 7582 (Wold et al. 2006), NGC 6240) additional faint extended structure is observed which contributes to less than $20 \%$ of the total MIR luminosity.

At $0.35^{\prime \prime}$ resolution with VISIR, the flux of all 36 Seyferts detected by Horst et al. (2006), Haas et al. (2007), and Horst et al. (2008) are dominated by an unresolved core. This observation is consistent with predictions of radiative transfer models for dust heated by an AGN. Intensity profiles of AGN dust models provide a typical MIR extent of 4 pc (FWHM, Fig. 22 in Siebenmorgen et al. 2004). This translates into an angular size of $0.08^{\prime \prime}$ at $10 \mathrm{Mpc}$ and is below the spatial resolution of VISIR. VLTI provides this spatial resolution but has insufficient sensitivity except for a handful of bright AGN, such as NGC 1068 and Circinus, which both have a small (few pc) flat central region of warm dust emission, coinciding with the positions of water maser emission for these galaxies (Jaffe et al. 2007). ESO's Extremely Large Telescope (ELT, $42 \mathrm{~m}$ ) will provide both sufficient sensitivity and 5 times higher spatial resolution than the VLT.

\subsection{Starburst - extended}

All five VISIR detected starbursts show extended nuclear emission of a few arcsec and structures on sub-arcsec scale. Intensity profiles, computed by radiative transfer models of dusty starbursts in which massive stars are distributed in a volume of $r=500 \mathrm{pc}$ radius, predict MIR extension of $\sim 160 \mathrm{pc}$ (FWHM, Fig. 22 in Siebenmorgen et al. 2004). At a distance of $100 \mathrm{Mpc}$, this translates into an angular size of $0.35^{\prime \prime}$, the resolution limit of VISIR.

A major result from IRAS was the discovery of a linear correlation between global measured far IR and radio emission of normal galaxies (Helou et al. 1985). The global picture is that IR emission traces the thermal emission of dust heated by stars, whereas radio observations detect primarily non-thermal emission from supernovae. Both infrared and radio emission are powered by early-type stars, albeit in different evolutionary states: before and during the main sequence phase they heat the dust and after the supernova explosion they produce copious synchrotron emission. This picture, however, cannot explain why the dispersion in this correlation is so small for entire galaxies spanning a wide range in parameters. The physical scale below which this correlation breaks is $\lesssim 100$ pc for the Milky Way (Boulanger $\&$ Perault 1988), but could not be probed on this scale in detail for external galaxies with existing infrared space missions, e.g. ISO: Xu et al. (1992) or Spitzer: Murphy et al. (2006), because of limited spatial resolution. Giuricin et al. (1994) found that the nuclear MIR emission of spiral galaxies is correlated with the radio emission when probed on scales of $5^{\prime \prime}$.

VLA archive data exist for three of our sources for which we can compare the radio - MIR correlation by means of radio contour overlays on the VISIR images. Limitations are: i) the spatial resolution (FWHM) of the radio maps of $\sim 0.6^{\prime \prime}$ compared to VISIR of $0.35^{\prime \prime}$ and ii) the astrometric uncertainty of our VLT data $\left(\sim 0.3^{\prime \prime}\right)$. In the following, we discuss the five objects individually:

\section{ESO126-G002}

In the VISIR image (Fig. 1), no central point source is detected. The MIR emission displays a circumnuclear ring structure quite similar to that found in NGC 7552 (Siebenmorgen et al. 2004). Striking emission clumps are observed $0.5^{\prime \prime} \mathrm{NW}$, and, a fainter one, $0.8^{\prime \prime} \mathrm{SE}$ of the center.

\section{Mkn 708 (NGC 2966)}

The VISIR image (Fig. 1) displays a central point source on top of a $4^{\prime \prime} \times 3^{\prime \prime}$ emission structure, extending West. The central core has a flux of $20 \mathrm{mJy}$, consistent with previous upper limits (Siebenmorgen et al. 2004). The extended component provides $90 \%$ of the total MIR luminosity and therefore we classify the nucleus as starburst dominated.

\section{ESO500-G034}

Using optical spectra, Hill et al. (1999) classified ESO500-G034 as an intermediate object between an AGN and starburst. Kewley et al. (2000) detected a compact radio core which argues for AGN rather than starburst heating of the dust. However, the radio core remained unconfirmed in images by Corbett et al. (2002). The astrometric precision of our observation does not enable us to locate the radio core in the VISIR image. The MIR image (Fig. 2) displays a $3^{\prime \prime} \times 4^{\prime \prime}$ nuclear extension. A dominant emission component is detected $0.5^{\prime \prime}$ to the NW from the nominal central position. It is resolved and contributes to less than $20 \%$ of the luminosity observed with VISIR. A second fainter clump is observed $0.5^{\prime \prime}$ South from the center. The beam size of the $6 \mathrm{~cm}$ VLA map is 1.6" (FWHM), insufficient to resolve the nuclear components. In the outer parts, the radio and MIR emission are correlated. Since we detect no dominant and unresolved luminosity component, we classify the MIR emission as being primarily starburst heated.

\section{Mkn 617}

This is a strongly interacting galaxy in a late stage of the merging process, which displays star forming regions observed in $\mathrm{H} \alpha$ (Dopita et al. 2002). X-ray spectroscopy suggests that Mkn 617 may harbor an obscured AGN (Risaliti et al. 2000). VLBI studies did not detect a compact radio core (Hill et al. 2001). The $11.7 \mu \mathrm{m}$ image acquired by Miles et al. (1996) provided a similar measurement of the size as the VISIR image (Fig. 2) but did not resolve the detailed structure of the central $1^{\prime \prime}$, where individual clumps on top of a diffuse component are detected. The radio emission follows the MIR morphology 

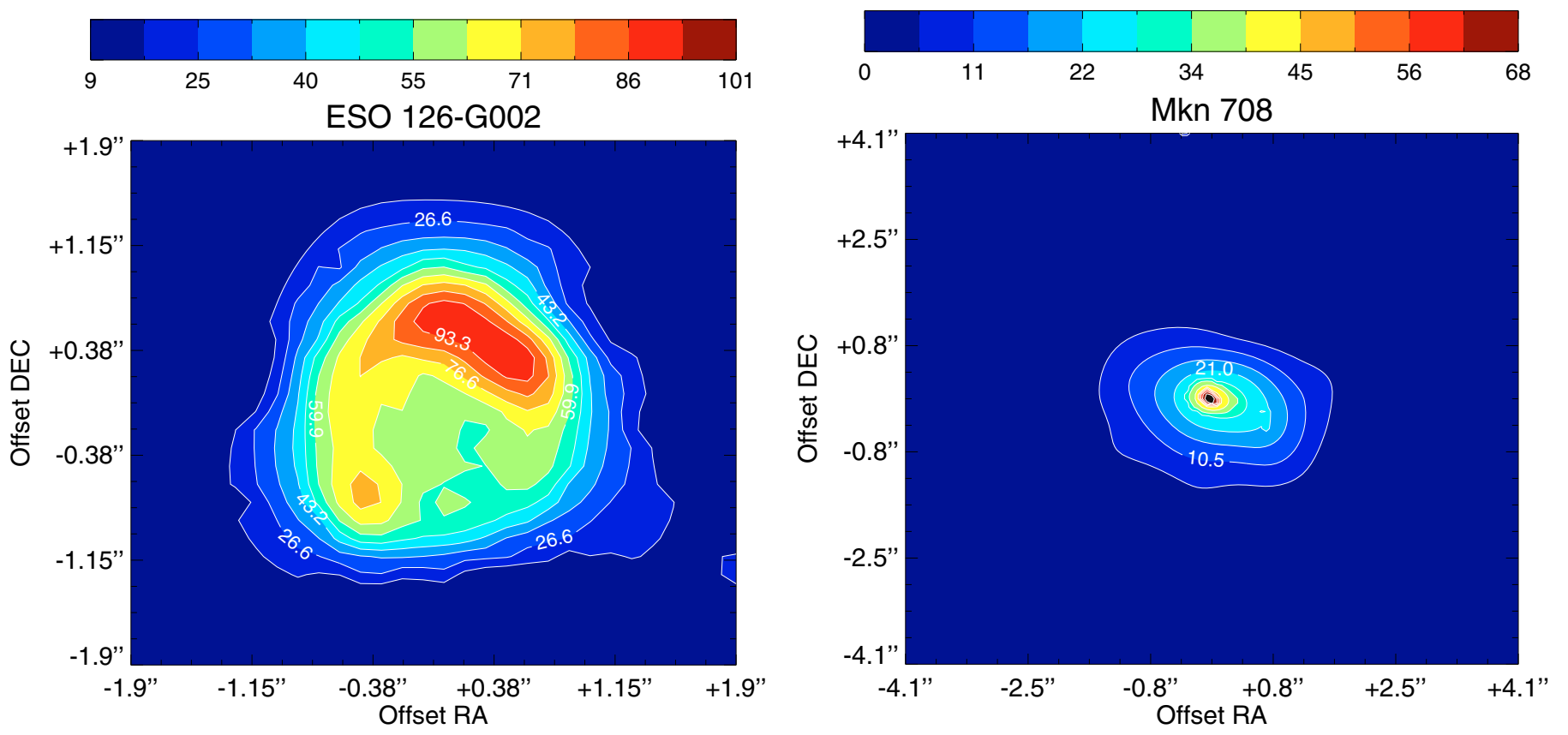

Fig. 1. VISIR image of ESO126-G002 (left) and Mkn 708 (right) in filter PAH2 (10.66-11.84 $\mu \mathrm{m}$ ) and spatial resolution of $\sim 0.35^{\prime \prime}$ (FWHM). North is up and East is to the left. Contours and colour look-up table in $\mathrm{mJy} / \operatorname{arcsec}^{2}$.

in the outer parts, but this correlation no longer exists in the central sub-arcsec region. However, differences may be related to insufficient spatial resolution in the VLA map of beam size of $0.6^{\prime \prime}$ (FWHM).

\section{NGC 1482}

IRAS observations indicated that NGC 1482 is a warm starburst galaxy with similar mass and size as M82. Its optical spectroscopic classification is that of a standard H II galaxy (Kewley et al. 2001). A multi-frequency radio continuum study was presented by Hota \& Saikia (2005). Strickland et al. (2004) found no sign of AGN activity in $\mathrm{H} \alpha$ and X-ray images. The soft X-ray revealed an outflow with super-wind morphology and strength similar to that observed in M82. The VISIR image (Fig. 2) reveals a double nucleus of $3^{\prime \prime}$ core separation that is well correlated with the $6 \mathrm{~cm}$ VLA map observed at $0.6^{\prime \prime}$ (FWHM) resolution.

\section{Comparison with SEDs and MIR spectra}

We compare the nuclear $11.3 \mu$ m flux with available MIR spectra and spectral energy distributions (SEDs) of the total galaxies. This comparison also involves radiative transfer calculations. To represent AGN, we choose NGC 3783 and NGC 4418 and for the starbursts Mkn 617 and NGC 1482 because of the completeness of available data ${ }^{1}$. The AGN are shown in Fig. 3 and the starbursts in Fig. 4. In agreement with earlier results (e.g.

\footnotetext{
1 Data of the Seyfert I NGC 3783 are from 2MASS, $3.6 \mu \mathrm{m}$ (Rieke 1978), MIR: VISIR $11.25 \mu \mathrm{m}$ (this work), Spitzer (Shi et al. 2006, IRS spectrum reprocessed here) and IRAS (Sanders et al. 2003). For the Seyfert II NGC 4418 the data are from NICMOS (Scoville et al. 2000), ISO (Spoon et al. 2001), IRAS (Sanders et al. 2003), $350 \mu \mathrm{m}$ (Benford 1999), $850 \mu \mathrm{m}$ (Dunne et al. 2000), and $1300 \mu \mathrm{m}$ (Albrecht et al. 2007). In addition, a MIR spectrum acquired by us at the ESO's $3.6 \mathrm{~m}$ with TIMMI2 between $8-13 \mu \mathrm{m}$ at spectral resolution of $\lambda / \Delta \lambda \sim 300$ and slit width of $3^{\prime \prime}$ is presented, our imaging at $10.4 \mu \mathrm{m}$ with TIMMI2 gives a $3 \sigma$ upper limit of $30 \mathrm{mJy}$. Evans et al. (2003) reported a $10.3 \mu \mathrm{m}$
}

Laurent et al. 2000; Brandl et al. 2006; Clavel et al. 2000; Weedmann et al. 2005), we find for our examples that i) PAH emission bands are strong in starbursts and weak or absent in AGN dominated galaxies; and ii) silicate absorption is strong in starburst and type II AGN.

The Seyfert II NGC 4418 exhibits strong extinction along a nuclear diameter of less than $50 \mathrm{pc}$ (FWHM, as estimated from the VISIR observations). Using radiative transfer models (Siebenmorgen et al. 2004), the SED can be fit by an AGN-heated optically-thick dust cloud of $A_{\mathrm{V}}=86 \mathrm{mag}$. Extinction is computed from the outer radius $r_{\text {out }}=450 \mathrm{pc}$ to the dust evaporation zone at $\sim 1 \mathrm{pc}$ and for simplicity a constant dust density profile is assumed. The model includes a central power-law heating source of $10^{11.8} L_{\odot}$.

The observed nuclear spectra of the starbursts are fitted by the SED model library of starburst nuclei by Siebenmorgen \& Krügel $(2007)^{2}$. Both starburst models predict a visual extinction of $A_{\mathrm{V}}=18 \mathrm{mag}$ which corresponds to $A_{10 \mu \mathrm{m}} \sim 1 \mathrm{mag}$; the dip

detection of $86 \mathrm{mJy}$ using MIRLIN, but this value is also inconsistent with the available Spitzer spectrum (Spoon et al. 2007). Otherwise various photometric data and spectra agree.

For the starbursts: data of Mkn 617 are from 2MASS (14" aperture), $11.7 \mu \mathrm{m}$ (Soifer et al. 2001), $11.25 \mu \mathrm{m}$ (this work) and Spitzer IRS (Brandl et al. 2006, reprocessed here), IRAS (Sanders et al. 2003), $160 \mu \mathrm{m}$ (Stickel et al. 2004), $350 \mu \mathrm{m}$ (Benford 1999), $450 \mu \mathrm{m}$ (Dunne et al. 2000), and $1300 \mu \mathrm{m}$ (Albrecht et al. 2007); and for NGC 1482 in the NIR from Hameed \& Devereux (2005), 2MASS (Spinoglio et al. 1995), $11.25 \mu \mathrm{m}$ (this work), Spitzer IRS (Kennicutt et al. 2003), IRAS (Sanders et al. 2003), $350 \mu \mathrm{m}$ (Chini et al. 1995), and $1300 \mu \mathrm{m}$ (Albrecht et al. 2007).

2 Model parameters are for Mkn 617: luminosity $L^{\text {tot }}=10^{11.2} L_{\odot}$, size $R=350$ pc, extinction $A_{\mathrm{V}}=18 \mathrm{mag}$, ratio of the luminosity of OB stars with hot spots to the total luminosity $L_{\mathrm{OB}} / L^{\text {tot }}=0.6$, density of hot spots $n^{\mathrm{hs}}=10^{4} \mathrm{~cm}^{-3}$; and for NGC 1482: $L^{\mathrm{tot}}=10^{10.2} L_{\odot}, R=350 \mathrm{pc}, A_{\mathrm{V}}=$ $18 \mathrm{mag}, L_{\mathrm{OB}} / L^{\text {tot }}=0.4, n^{\mathrm{hs}}=10^{4} \mathrm{~cm}^{-3}$, and PAH abundance of $10 \%$ of $\mathrm{C}$, which is a factor of 5 larger than for other elements of the SED model library. To fit the data below $5 \mu \mathrm{m}$, the starburst models of Mkn 617 and NGC 1482 are supplemented with an old stellar population represented by a black body of $T=3000 \mathrm{~K}$. 

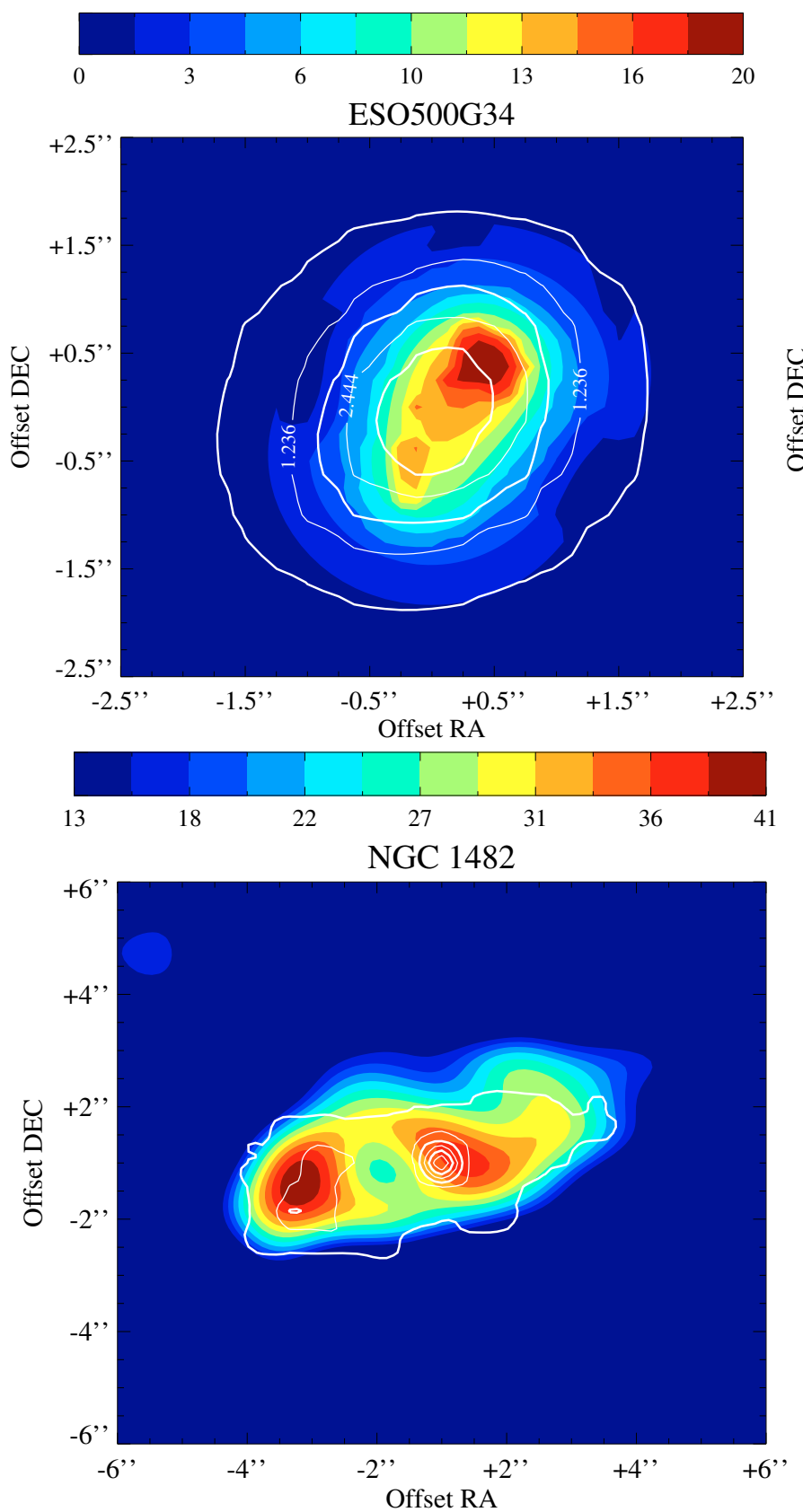
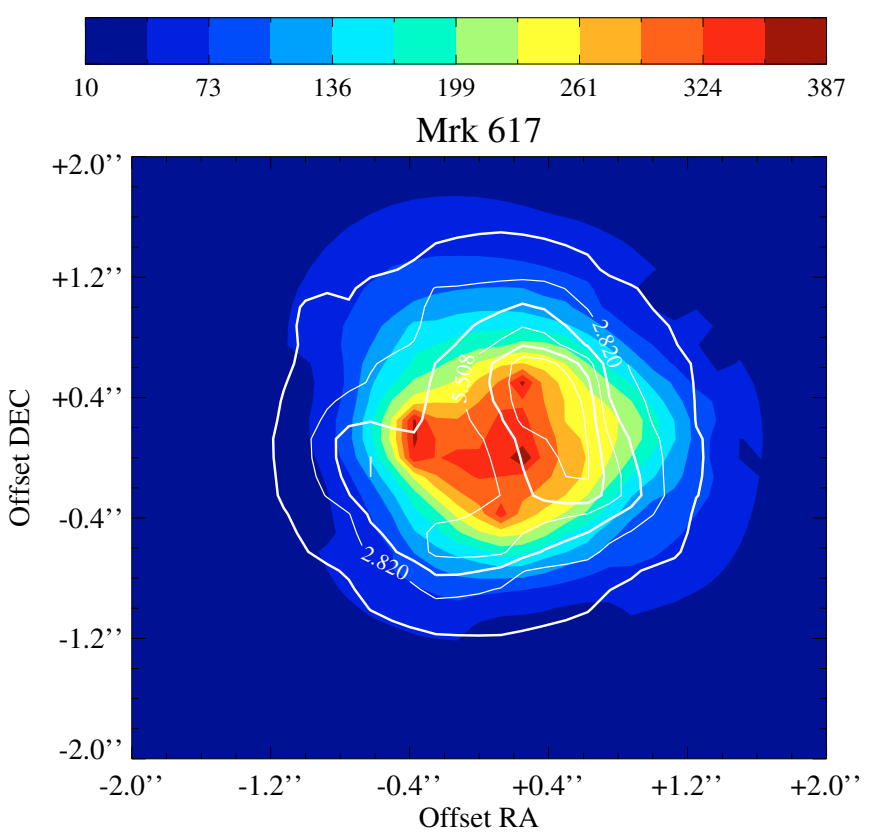

Fig. 2. VISIR images (colour look-up table in $\mathrm{mJy} / \operatorname{arcsec}^{2}$ ) overlaid with VLA $6 \mathrm{~cm}$ radio contours. VISIR data are in filter PAH2 (10.66$11.84 \mu \mathrm{m}$ ) at spatial resolution of $\sim 0.35^{\prime \prime}$ (FWHM). Radio contours of ESO500-G034 (beam 1.6", FWHM) are from 0.632 increased in steps of 0.604 , that of Mkn $617\left(0.6^{\prime \prime}\right)$ from 1.48 in steps of 1.34 and NGC $1482\left(0.6^{\prime \prime}\right)$ from 0.618 in steps of 0.62 (mJy/arcsec $\left.{ }^{2}\right)$, respectively. North is up and East is to the left.

at $10 \mu \mathrm{m}$ is due to silicate absorption and is not a blend of PAH emission features. The nuclear starburst emission spectrum is not representative of the total emission of the galaxy; this is most evident at long wavelengths (Fig. 4), for which most of the FIR luminosity is due to large extended cold or even very cold dust emission (e.g. Siebenmorgen et al. 1999). On the other hand, most of the emission up to $200 \mu \mathrm{m}$ in AGN, such as NGC 4418, originates in the central $500 \mathrm{pc}$ region.

\section{Nuclear MIR surface brightness}

So far, we have seen that optically classified starburst nuclei are resolved by VISIR, while in AGN, an unresolved core dominates the MIR flux. In addition to the qualitative morphology criterion, it is desirable to quantify the effective gain reached, for instance, by VISIR when trying to distinguish between AGN and SB. Therefore, we consider the nuclear MIR surface brightness $S$ of our distance limited $(100 \mathrm{Mpc})$ sample as shown in Fig. 5. For resolved sources without dominant unresolved core, an area containing $80 \%$ of the flux is used (Table 1). For unresolved sources and non-detections, an aperture of radius $0.35^{\prime \prime}$ is adopted, corresponding to lower and upper limits of $S$, respectively. The AGN are supplemented with 9 sources $(<100 \mathrm{Mpc})$ observed with VISIR by Horst et al. (2006, 2008). The starbursts are supplemented with 4 resolved objects (Mrk1093, NGC 3256, NGC 6000 and NGC 7552) observed with ESO $3.6 \mathrm{~m} / \mathrm{TIMMI} 2$ 

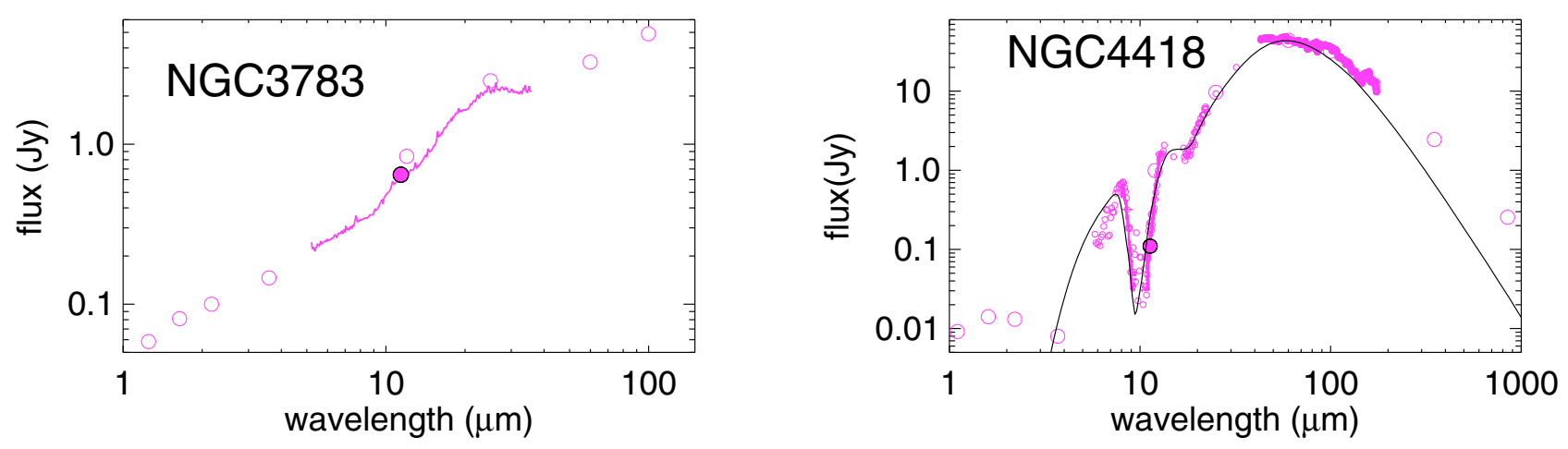

Fig. 3. SED of the Sy I galaxy NGC 3783 (left) and the Sy II galaxy NGC 4418 (right). Photometry is marked with circles and spectra with magenta lines. For both Seyferts the nuclear VISIR photometry closely resembles that of the total galaxy. This appears to hold also at FIR-mm wavelengths, as inferred from the radiative transfer model (black solid line).
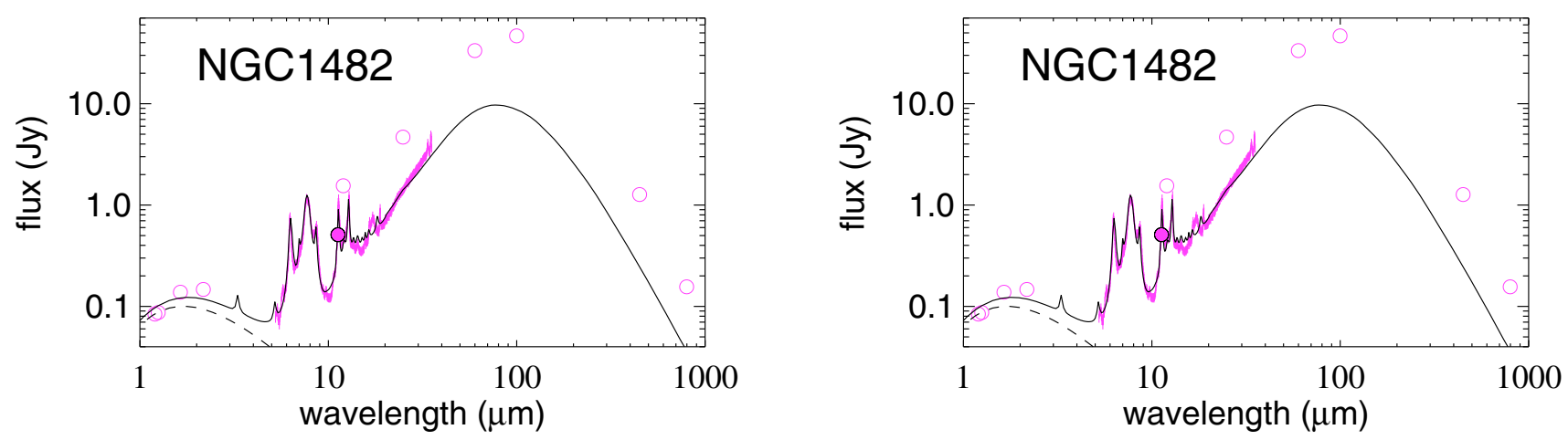

Fig. 4. SED of the starburst galaxies Mkn 617 (left) and NGC 1482 (right). Photometry is marked with circles and spectra with magenta lines. While for Mkn 617 the nuclear VISIR photometry closely resembles that of the total galaxy, the nucleus of NGC 1482 contains at most $25 \%$ of the total MIR flux. As inferred from the radiative transfer model (black solid line) the difference between nucleus and total galaxy remains (NGC 1482) and increases (Mkn 617) towards FIR-mm wavelengths. The visual extinction is in both starburst models 18 mag so that the silicate band at $10 \mu \mathrm{m}$ is seen in absorption.

by Siebenmorgen et al. (2004). For comparison, (ultra)-luminous IR galaxies observed by Keck/MIRLIN (Soifer et al. 2000) and Gemini/T-ReCS (Alonso-Herrero et al. 2006; Mason et al. 2007) are shown, although these sources are at larger distances; results are:

1) At the spatial resolution of VISIR, AGN and SB are clearly separated using the nuclear MIR surface brightness $S$ as a criterion. AGN are observed at $S \gg 10^{4} L_{\odot} / \mathrm{pc}^{2}$, whereas most of the SB are observed to be a factor of 3-10 fainter at $S \sim 10^{3} L_{\odot} / \mathrm{pc}^{2}$.

2) A distinction between $A G N$ and $S B$ is not possible using MIR images acquired by $4 \mathrm{~m}$ class telescopes, even when adopting the theoretical diffraction limit of 0.7" FWHM (small gray symbols in Fig. 5, data as compiled by Haas et al. 2007). Since the PSF width is twice as large, it becomes more difficult to resolve starbursts and the surface brightness of unresolved sources is reduced.

3) SB populate a surface brightness range around and below that of Orion $\left(1200 \lesssim S \lesssim 4 \times 10^{4} L_{\odot} / \mathrm{pc}^{2}\right){ }^{3}$

\footnotetext{
3 We have analysed VISIR archive data of the Orion hot core. Images available are of $0.075^{\prime \prime}$ pixel scale providing a $18^{\prime \prime} \times 18^{\prime \prime}$ field $\left(\sim 0.002 \mathrm{pc}^{2}\right)$. In this area, the mean MIR surface brightness of the Orion
}

4) The AGN - SB separation is consistent with predictions of radiative transfer models for starburst galaxies (dashed and dotted lines in Fig. 5).

5) The AGN - SB separation appears to hold when comparing (U)-LIRGs observed by Keck/MIRLIN and Gemini/T-ReCS (data from Soifer et al. 1999, 2000, 2001; Alonso-Herrero et al. 2006; Mason et al. 2007).

6) Normal, inactive galaxies have lower nuclear MIR surface brightness than SB.

The high spatial resolution imaging provides an important diagnostic tool to determine the activity type of galactic-nuclei, only limited by the diffraction of the telescope. Such imaging with $8 \mathrm{~m}$ class telescopes can be used to distinguish between AGN and SB activity out to distances of $\sim 100 \mathrm{Mpc}$. Indeed, all of our detected starbursts show resolved structures. On the other hand, all unresolved sources of nuclear MIR surface brightness $S>20000 L_{\odot} / \mathrm{pc}^{2}$ are found in galactic nuclei with optical

hot core is $\sim 40800 L_{\odot} / \mathrm{pc}^{2}$. Werner et al. (1976) found in a $5^{\prime}$ far IR map a total luminosity of $1.2 \times 10^{5} L_{\odot}$ emerging from a region of $0.4 \mathrm{pc}^{2}$. This converts to a MIR surface brightness $S \sim 1200 L_{\odot} / \mathrm{pc}^{2}$, where we scaled the IR luminosity to that in the VISIR PAH2 filter applying $\log L_{11} \sim 1.06 \log (L)-2.7$ (relation derived using template spectrum). 


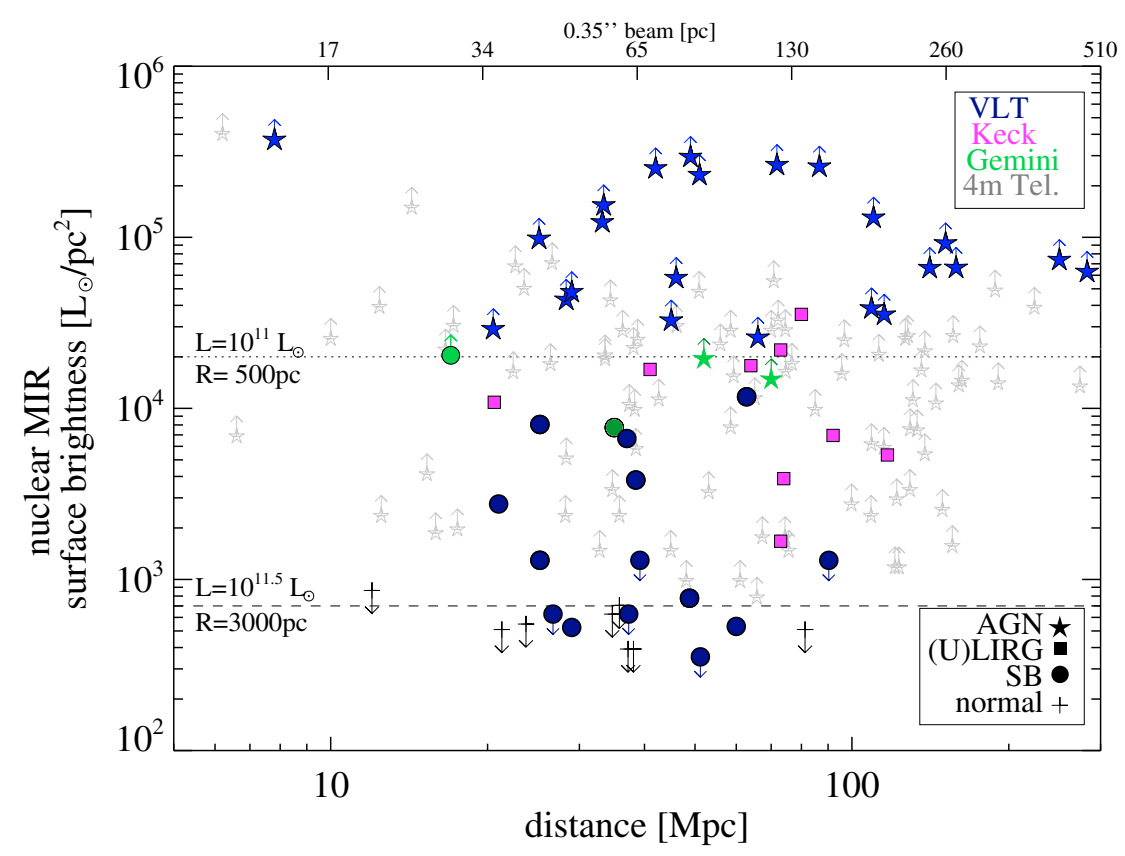

Fig. 5. Nuclear MIR surface brightness versus distance. Large symbols mark data from VLT (blue): Haas et al. (2007), Horst et al. (2006, 2008), this work; Keck (magenta): Soifer et al. (1999, 2000, 2001); Gemini (green): Alonso-Herrero et al. (2006), Mason et al. (2007); small symbols from $4 \mathrm{~m}$ class telescopes (gray): Haas et al. (2007). AGN (stars) have $S>20000 L_{\odot} / \mathrm{pc}^{2}$ when observed with $8 \mathrm{~m}$ class telescopes, starburst and (U)LIRGs are below (with the exception of VV114A which may probably habour an AGN). Normal galaxies (+) are not detected. The horizontal dotted and long-dashed lines give the surface brightness computed with starburst models by Siebenmorgen \& Krügel (2007) for given total luminosity $L$ and where stars and dust are distributed in a $A_{\mathrm{V}}=18$ mag nucleus of radius $R$.

identified AGN signatures and we classify their MIR emission to be AGN driven. For example, NGC1097, which displays an unresolved core $(<45 \mathrm{pc})$ in the MIR and a surface brightness of $S=10^{4} L_{\odot} / \mathrm{pc}^{2}$, is not AGN driven but heated by an unresolved star cluster (Mason et al. 2007). The VISIR measured value of $S$ for Cen A is consistent with that found using Gemini (Radomski et al. 2008). Other caveats have to be mentioned for radio loud AGN where synchrotron radiation may dominate the nuclear MIR emission, e.g. M87 (Whysong \& Antonucci 2004; Perlman et al. 2007) or low luminosity AGN, such as LINERs, which are not investigated here.

Acknowledgements. This research has made use of the NASA/IPAC Extragalactic Database (NED) and observations with the Spitzer Space Telescope which is operated by the Jet Propulsion Laboratory, California Institute of Technology, under contract with the National Aeronautics and Space Administration. M.H. is supported by the Nordrhein-Westfälische Akademie der Wissenschaften. We thank the anonymous referee for detailed critical suggestions.

\section{References}

Albrecht, M., Krügel, E., \& Chini, R. 2007, A\&A, 462, 575 Alonso-Herrero, A., Colina, L., Packham, C., et al. 2006, ApJ 652, L83 Benford, D. J. 1999, Thesis, California Institute of Technology Boulanger, F., \& Perault, M. 1988, ApJ, 330, 964

Brandl, B. R., Bernard-Salas, J., Spoon, H.W.W. et al. 2006, ApJ, 653, 1129

Buchanan, C. L., Gallimore, J. F., O’Dea, C.P., Baum, S. A., \& Axon, D. J. 2006, ApJ, 132, 401

Chini, R., Krügel, E., \& Kreysa, E. 1992, A\&A, 266, 177

Chini, R., Krügel, E., Lemke, R., \& Ward-Thompson, D. 1995, A\&A, 295, 317

Clavel, J., Schulz, B., Altieri, B., et al. 2000, A\&A, 357, 839

Corbett, E. A., Norris, R. P., Heisler, C. A., et al. 2002, ApJ, 564, 650

Dopita, M. A., Pereira, M., Kewley, L. J., \& Capaccioli, M. 2002, ApJS, 143, 47

Dunne, L., Eales, S., Edmunds, M., et al. 2000, MNRAS, 315, 115

Evans, A.S., Becklin, E.E., Scoville, N.Z., et al. 2003, AJ, 125, 2341
Galliano, E., Alloin, D., Pantin, E., Lagage, P. O., \& Marco, O. 2005a, A\&A, 438,803

Galliano, E., Alloin, D., Pantin, E., \& Lagage, P. O. 2005b, MNRAS, 363, L1

Genzel, R., Lutz, D., Sturm, E., et al. 1998, ApJ, 498, 579

Gorjian, V., Werner, M.W., \& Jarrett, T.H. 2004, ApJ, 605, 156

Giuricin, G., Tamburini, L., Mardirossian, F., Mezzetti, F., \& Monaco, P. 1994, ApJ, 427, 202

Hameed, S., \& Devereux, N. 2005, AJ, 129, 2597

Hao, L., Spoon, H. W. W., Sloan, G. C., et al. 2005, ApJ, 625, L75

Haas, M., Siebenmorgen, R., Pantin, E., et al. 2007, A\&A, 473, 369

Helou, G., Soifer, B. T., \& Rowan-Robinson, M. 1985, ApJ, 298, L7

Hill, T. L., Heisler, C. A., Sutherland, R., \& Hunstead, R. W. 1999, AJ, 117, 111

Hill, T. L., Heisler, C. A., Norris, R. P., Reynolds, J. E., \& Hunstead R. W. 2001, AJ, 121,128

Horst, H., Smette, A., Poshak, G., \& Duschl, W. J. 2006, A\&A, 457, 17

Horst, H., Poshak, G., Smette, A., \& Duschl, W. J. 2008, A\&A, 479, 389

Hota, A., \& Saikia, D. J. 2005, MNRAS, 356, 998

Jaffe, W., Raban, D., Röttgering, H., Meisenheimer, K., \& Tristram, K. 2007, in The Central Engine of Active Galactic Nuclei, ASP Conference Series, 373, 439

Kennicutt, R. C. Jr., Armus, L., Bendo, G., et al. 2003, PASP, 115, 928

Kewley, L. J., Heisler, C. A., Dopita, M. A., et al. 2000, ApJ, 530, 704

Kewley, L. J., Heisler, C. A., Dopita, M. A., \& Lumsden, S. 2001, ApJS, 132, 37

Krabbe, A., Böker, T., \& Maiolino, R. 2001, ApJ, 557, 626

Krügel, E., Chini, R., \& Steppe, H. 1990, A\&A, 229, 17

Lagage, P. O., Pel, J., Authier, M., et al. 2004, Messenger, 117, 12

Laurent, O., Mirabel, I. F., Charmandaris, V., et al. 2000, A\&A, 359, 887

Maiolino, R., Ruiz, M., Rieke, G. H., \& Keller, L.D. 1995, ApJ, 446, 561

Mason, R. E., Levenson, N. A., Packham, C., et al. 2007, ApJ, 659, 249

Miles, J. W., Houck, J. R., Hayward, T. L., \& Ashby, M.L.N. 1996, ApJ, 465, 191

Mulchaey, J. S, Wilson, A. S., \& Tsvetanov, Z. 1996, ApJS, 102, 309

Murphy, E. J., Braun, R., Helou, G., et al. 2006, ApJ, 638, 157

Pantin, E., Lagage, P.-O., Claret, A., et al. 2005, Messenger, 119, 25

Perlman, E., Mason, R. E., Packham, C., et al. 2007, ApJ, 663, 808

Rieke, G. H. 1978, ApJ, 226, 550

Rieke, G. H., Lebofsky, M., \& 1978, ApJ, 220, L38

Risaliti, G., Gilli, R., Maiolino, R., Salvati, M. 2000, A\&A, 357, 13

Roche, P., Aitken, D., Smith, C., \& Ward, M. 1991, MNRAS, 248, 606

Roche, P. F., Packham, C., Telesco, C. M., et al. 2006, MNRAS, 367, 1689 
Radomski, J. T., Packham, C., Levenson, N. A., et al. 2008 [arXiv: 0802. 4119] Sanders, D. B., Mazzarella, J. M., Kim, D.-C., et al. 2003, AJ, 126, 1607 Scoville, N. Z., Evans, A. S., Thompson, R., et al. 2000, AJ, 119, 991

Shi, Y., Rieke, G. H., Hines, D. C., et al. 2006, ApJ, 653, 127

Siebenmorgen, R., \& Krügel, E. 2007, A\&A, 461, 445

Siebenmorgen, R., Krügel, \& E., Chini 1999, A\&A, 351, 495

Siebenmorgen, R., Krügel, E., \& Spoon, H. W. W. 2004, A\&A, 414, 123

Siebenmorgen, R., Haas, M., Krügel E., \& Schulz, B. 2005, A\&A, 436, L5

Soifer, B. T., Neugebauer, G., Matthews, K., et al. 1999, ApJ, 513, 207

Soifer, B. T., Neugebauer, G., Matthews, K., et al. 2000, AJ, 119, 509

Soifer, B. T., Neugebauer, G., \& Matthews, K. 2001, AJ, 122, 1213

Spinoglio, L., Malkan, M. A., Rush, B., Carrasco, L., \& Recillas-Cruz, E. 1995, ApJ, 453, 616

Spoon, H. W. W., Keane, J. V., Tielens, A. G. G. M., et al. 2001, A\&A, 365, L353
Spoon, H. W. W., Moorwood, A. F. M., Lutz, D., et al. 2004, A\&A, 414, 873 Spoon, H. W. W., Marshall, J. A., Houck, et al. 2007, ApJ, 654, L49

Starck, J. L., \& Murtagh, F. 2002, Astronomical image and data analysis (Berlin: Springer) ISBN 3540428852

Stickel, M., Lemke D., Klaas U., et al. 2004, A\&A, 422, 39

Strickland, D. K., Heckman, T. M., Colbert, E. J. M., Hoopes, C. G., \& Weaver, K. A. 2004, ApJS, 151, 193

Sturm, E., Lutz, D., Verma, A., et al. 2002, A\&A, 393, 821

Sturm, E., Rupke, D., Contursi, A., et al. 2006, ApJ, 653, L13

Thean, A., Pedlar, A., \& Kukula, M. J. 2000, MNRAS, 314, 573

Weedman, D. W., Hao, L., Higdon, S. J. U., et al. 2005, ApJ, 633, 706

Werner, M.W., Gatley, I., Harper, D. A., et al. 1976, ApJ, 204, 420

Wold, M., Lacy, M., Käufl, H. U., \& Siebenmorgen, R. 2006, A\&A, 460, 449

Whysong, D., \& Antonucci, R. R. 2004, ApJ, 602, 116

Xu, C., Klein, U., Meinert, D., et al. 1992, A\&A, 257, 47 